\title{
Representing Caused Motion in Embodied Construction Grammar
}

\author{
Ellen K. Dodge \\ International Computer Science Institute \\ Berkeley, CA, USA 94704 \\ edodge@icsi.berkeley.edu
}

\author{
Miriam R. L. Petruck \\ International Computer Science Institute \\ Berkeley, CA, USA 94704 \\ miriampdicsi.berkeley.edu
}

\begin{abstract}
This paper offers an Embodied Construction Grammar (Feldman et al. 2010) representation of caused motion, thereby also providing (a sample of) the computational infrastructure for implementing the information that FrameNet has characterized as Caused motion ${ }^{1}$ (Ruppenhofer et al. 2010). This work specifies the semantic structure of caused motion in natural language, using an Embodied Construction Grammar analyzer that includes the semantic parsing of linguistically instantiated constructions. Results from this type of analysis can serve as the input to NLP applications that require rich semantic representations.
\end{abstract}

\section{Introduction}

Computational linguistics recognizes the difficulty in articulating the nature of complex events, including causation, while understanding that doing so is fundamental for creating natural language processing (NLP) systems (e.g. Girju 2003, Quang et al. 2011), and more generally for other computational techniques (Guyon et al. 2007, Friedman et al. 2000). Also, although insightful, linguistic analyses of causation are insufficient for systems that require drawing the inferences that humans draw with ease. Such systems must incorporate information about the parameters that support drawing these inferences. Embodied Construction Grammar (ECG) provides the computational and representational apparatus for capturing what language expresses.

FrameNet (FN) frames capture event structure in terms of the participants that play a role in that event. ECG provides a means for the automatic identification of frames and frame roles ex-

\footnotetext{
${ }^{1}$ Courier New font indicates a FrameNet frame; and small CAPS indicate a FE in a FN frame.
}

pressed in any given sentence. Here, we focus on a pair of sentences that illustrate the rich meanings and relations characterized in $\mathrm{FN}$ frames as represented in ECG.

This paper is organized as follows: Section 2 includes background information on FrameNet (FN) and ECG; Section 3 describes the FN treatment of Caused motion, and the ECG representation of the CauseMotion schema, which constitutes the meaning block of the Cause_motion construction; Section 4 summarizes the ECG analysis of motion and caused motion example sentences; and Section 5 discusses new directions for future work with ECG representations of information structured in FrameNet frames (http://framenet.icsi.berkeley.edu).

\section{Background}

Chang et al. (2002) constitutes the first effort to represent the prose description of the information that FN has defined in semantic frames in formal terms. The work provided an ECG representation of FN's (then) Commerce frame, showing the perspicuity of doing so to account for linguistic perspective, and ultimately useful in translating FN information into a representation needed for event simulation (Narayanan 1997). Building on Chang et al. (2002), this paper focuses on the analysis and representation of the meanings of sentences describing different kinds of motion events, using a set of related semantic frames. Before detailing the examples that illustrate the analysis and representation, we offer a very brief overview of FN and ECG.

\subsection{FrameNet}

The FrameNet knowledge base holds unique information on the mapping of meaning to form via the theory of Frame Semantics (Fillmore and Baker 2010), at the heart of which is the semantic frame, i.e. an experience-based schematization of the language user's world that allows inferences about participants and objects in and across situations, states of affairs, and events. FN 
has characterized nearly 1,200 frames, more than 12,740 lexical units (LUs), where a lexical unit is a pairing of a lemma and a frame, and approximately 200,000 manually annotated example sentences that illustrate usage of each LU.

A FN frame definition includes a prose description of a prototypical situation, along with a specification of the frame elements (FEs), or semantic roles, that uniquely characterize that situation. For example, FN has defined Motion as a situation in which a THEME starts out at a SOURCE, moves along a PATH, and ends up at a GOAL. $^{2}$ Example (1) illustrates FN's analysis of SLIDE, one of many LUs, in the Motion frame, also indicating which constituents in the sentence are the linguistic realizations of the FEs THEME and GOAL.

(1) [The envelope THEME] SLID [into the mailbox $\left.{ }_{\text {GOAL }}\right]$.

\subsection{Embodied Construction Grammar}

An ECG grammar (Feldman et al. 2010) consists of structured lattices of two basic primitives: schemas and constructions. As with other forms of construction grammar, a key tenet of ECG is that each construction is a form-meaning pair. Constructional meaning is represented using schemas, which are analogous to FN frames. Each schema includes several roles (comparable to FN's FEs), and specifies various types of constraints on its roles. Thus, the ECG formalism provides the means for representing frame structure and relations in a precise and computationally tractable manner.

Crucially, our computationally implemented system (Bryant 2008) uses an ECG grammar for sentence interpretation and produces construction-based semantic parses. The Constructional Analyzer utilizes form and meaning information in the constructional analysis of a given sentence. Thus, constructional analysis is not just a form match; importantly, it is a meaning match as well. The output of the analysis is a semantic specification, a meaning representation in the form of schemas, roles, bindings, and role-filler information. Constructional analysis is part of a larger model of language understanding, in which the semantic specification, in conjunction with discourse and situational context, serves as an input for a simulation process, which fleshes

\footnotetext{
${ }^{2}$ FN also describes another scenario for Motion, not included here for space-saving reasons.
}

out and supports further inferences about the relatively schematic meaning specified in the text.

Among the potential applications for such deep semantic analysis are question-answering tasks, information extraction, and identifying different political viewpoints. Each task has its own requirements in terms of constructions and semantic representations. This paper illustrates a computationally implemented means for determining the frames and frame roles in a given sentence, as well as the particular entities that link to those roles.

Figure 1 shows the ECG formalism that represents the semantic structure of FN's Motion frame, where the MotionPath schema specifies the structural relationships among the participants (FEs) in FN's Motion frame.

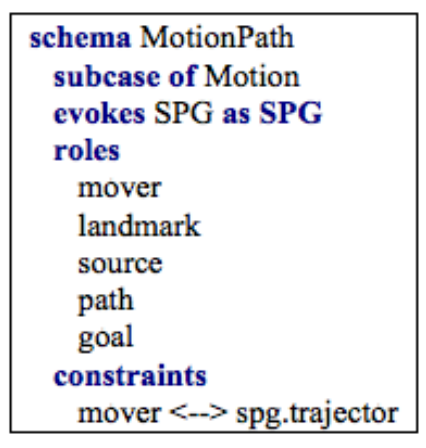

Figure 1: MotionPath Schema

ECG defines MotionPath as a subcase of the very general Motion schema; as a child of the Motion schema, MotionPath inherits the structure of Motion, the latter including a mover role. Also, MotionPath evokes a Source-Path-Goal (SPG) schema, which includes the roles source, path, and goal, for the initial, medial, and final locations, respectively, of a trajector. MotionPath also includes a constraint that the mover is bound to the trajector role.

A construction is a pairing between form and meanng, the ECG representation of which consists of a form block and a meaning block. To illustrate, in Figure 2, which shows the simple lexical construction Slid_Past, the form block indicates the orthographic form associated with the lexical construction. The form constraint indicates that the constraint applies to the form of the construction (self.f.orth <-- "slid"), where .orth indicates that the form is a text string, in this case "slid". ECG represents constructional meaning using schemas; in the Slid_Past construction, (Figure 2), the meaning is identified with the MotionPath schema (as shown in Figure 1). Constructions also define relations to other 
constructions in the grammar. For instance, Slid_Past is a subcase of a more general PastTense construction. The PastTense construction is one of several general verb conjugation constructions in the grammar, each of which captures general facts about tense and aspect associated with different verb conjugation forms. For example, all past tense verbs, including slid (an instantiation of Slid_Past), use PastTense.

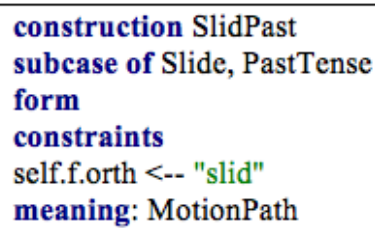

Figure 2: Slid_Past Construction

\section{Caused_motion}

This section describes FN's characterization of Caused motion and provides the ECG representation of the CausedMotion schema, i.e., the meaning of the Cause_motion construction.

\subsection{FrameNet}

FrameNet characterizes Caused_motion as a situation in which an AGENT causes a THEME to undergo translation motion, where the motion also may always be described with respect to a SOURCE, PATH, and GOAL. ${ }^{3}$ Example (2) shows the FN analysis with SLIDE as the target verb in Caused motion, marking the constituents that fill the AGENT, THEME, and GOAL roles.

(2) [Smedlap AGENT] SLID [the envelope THEME] into the mailbox ${ }_{\mathrm{GOAL}}$ ].

Note that whereas FN's Caused_motion frame has an AGENT FE, Motion does not.

\begin{tabular}{|c|c|l|}
\hline FrameNet Frame & Frame Elements & Example Sentence \\
\hline Motion & $\begin{array}{c}\text { THEME } \\
\text { SOURCE } \\
\text { PATH } \\
\text { GOAL }\end{array}$ & $\begin{array}{l}\text { The envelope slid } \\
\text { into the mailbox. }\end{array}$ \\
\hline \multirow{3}{*}{ Caused_motion } & $\begin{array}{c}\text { AGENT } \\
\text { THEME } \\
\text { SOURCE } \\
\text { PATH } \\
\text { GOAL }\end{array}$ & $\begin{array}{l}\text { Smedlap slid the } \\
\text { envelope into the } \\
\text { mailbox. }\end{array}$ \\
\hline
\end{tabular}

Table 1: FN's Motion and Caused_motion

\footnotetext{
${ }^{3}$ As with Motion, FN also defines another scenario for Caused_motion, specifically one with a CAUSE FE, excluded here because of space limitations.
}

\subsection{Embodied Construction Grammar}

The ECG representation of CauseMotion, given in Figure 3, is a complex schema that combines the structure of causation with that of translational motion. The causal structure is supplied by defining CauseMotion as a subcase of a more general CauseEffect schema, which includes roles for a causal agent and an affected entity. Also, CauseMotion specifies that the effect is translational motion, indicated with the addition of a type constraint that identifies the effect with the MotionPath schema.

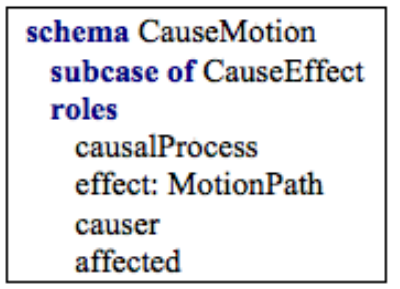

Figure 3: CauseMotion Schema

Additional constraints bind the mover role of MotionPath (Figure 1) to the affected role of CauseMotion. Thus, the ECG mover (i.e. the FE THEME) is bound to the affected role; and the motion itself is bound to the effect. ECG uses the CauseMotion schema to analyze sentences such as Example (2), an instance of the Cause_motion construction, a summary of which follows below in Section 4.

\section{ECG for Linguistic Analysis}

Here, we provide an overview of the ECG analysis process for the examples discussed above.

A basic tenet of construction grammar is that each sentence instantiates multiple constructions. Bryant's (2008) computationally implemented Constructional Analyzer uses an ECG grammar to determine the set of constructions that best-fit a given sentence. ${ }^{4}$ The assessment of "best-fit" takes both syntactic and semantic information into account. Constructional unification requires compatibility between unifying elements. Unification tests the compatibility of the constraints that these constructions specify, and leads to various bindings. The analyzer produces a SemSpec, i.e. a semantic specification of the sentence, that is, a meaning representation in the form of a network of schemas, with bindings between schema roles and fillers of these roles.

\footnotetext{
${ }^{4}$ ECG Workbench provides an interface to the analyzer, and allows the viewing of analysis results.
} 
To illustrate the analysis process, we revisit Example (1) The envelope slid into the mailbox, which instantiates some of the following lexical and grammatical constructions:

- Lexical constructions for slid, nouns (envelope, mailbox), and determiners (the)

- NP constructions (the envelope, the mailbox)

- Declarative construction, a clause-level construction spanning the sentence as a whole.

In what follows, we define and explain the constructions in Example (1) that are crucial for the present analysis of Motion and CausedMotion. Specifically, the Active_Motion_Path construction (Figure 4) is a sub-case of a more general Argument Structure construction (Goldberg 1995, Dodge 2010). Argument Structure (A-S) constructions specify general patterns of role expression associated with the description of basic experiences, such as those involving motion, perception, object transfer, actions and/or causation. Schemas represent each type of experience and include roles for the relevant participant(s). A-S constructions include one or more constituent constructions, specified within their constructional block. All A-S constructions include a verb constituent (V: Verb); here, note that the Active_Motion_Path construction also contains a prepositional phrase constituent (PP), constrained to be of type Path-PP, a general construction that identifies its meaning with the SPG schema. The form block specifies ordering constraints: the $\mathrm{V}$ constituent comes before the PP constituent. The meaning block specifies that the construction's meaning is identified with the MotionPath schema, indicating that the construction describes translational motion events. Constraints within the meaning block specify how the meaning of the construction as a whole composes with the meanings of its constituents. As is usually the case, the ' $\mathrm{V}$ ' constituent shares the same schematic meaning as the A-S construction. These constraints indicate that the A-S construction will unify with verbs that identify their meaning with the MotionPath schema, as in the motion verbs roll, slip, bounce, etc, along with slide. Thus, this A-S construction captures a particular valence pattern associated with several semantically similar verbs.

A further meaning constraint indicates that the meaning of the PP constituent serves to elaborate the SPG schema that forms part of the meaning of the MotionPath schema. That is, whichever specific PP serves as a constituent in a given example will supply more information about the particular path of motion the mover is following. Additionally, the mover role of MotionPath is bound to the profiledParticipant role (i.e., the semantic correlate of the role expressed in the sentence's subject slot), and the meaning of the construction as a whole binds to an eventProcess role, which indicates the type of event that this A-S construction describes.

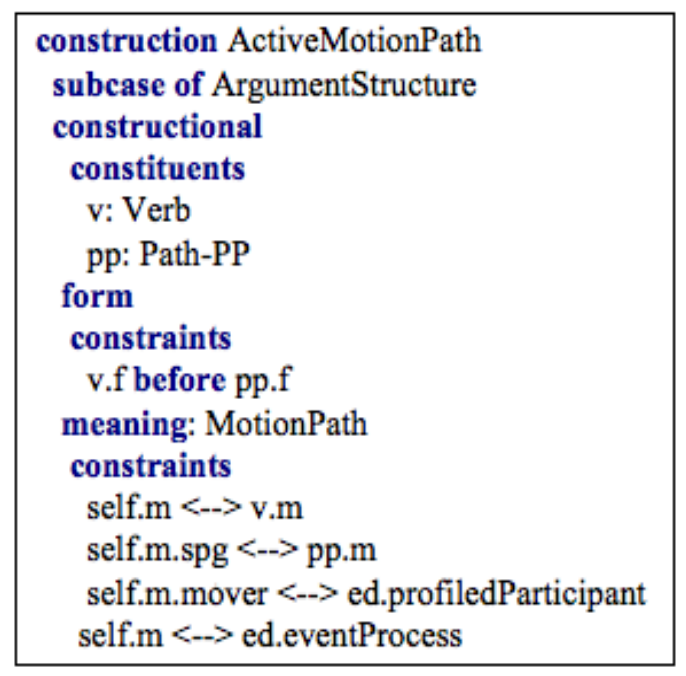

Figure 4: Active_Motion_Path Construction

Constraint-based unification of the instantiated construction produces a SemSpec that includes the following information: (1) a MotionPath schema is co-indexed with the 'eventProcess' role, indicating that the sentence describes an event of translational motion; (2) the meaning of the envelope is co-indexed with the profiledParticipant role, the mover of MotionPath, and the trajector of SPG, indicating that this object is the semantic subject of the sentence, and that it is the entity that moves and changes location with respect to a landmark; and (3) the meaning of the mailbox is co-indexed with the landmark of SPG, and the boundedObject role of a BoundedObject schema. The source of SPG is bound to the exterior role of boundedObject. The goal of SPG is bound to the interior of boundedObject. Together, these bindings indicate that the mailbox is conceptualized as a bounded object or container, the envelope's initial location (source) is outside of the mailbox, and its final location is inside.

Having analyzed a sentence about motion, we return to our example of caused motion: (2) Smedlap slid the envelope into the mailbox. This sentence instantiates many of the same constructions as does Example (1). The key difference between the two is the A-S construction, here the 
Active_Transitive_Caused_Motion construction, shown below in Figure 5.

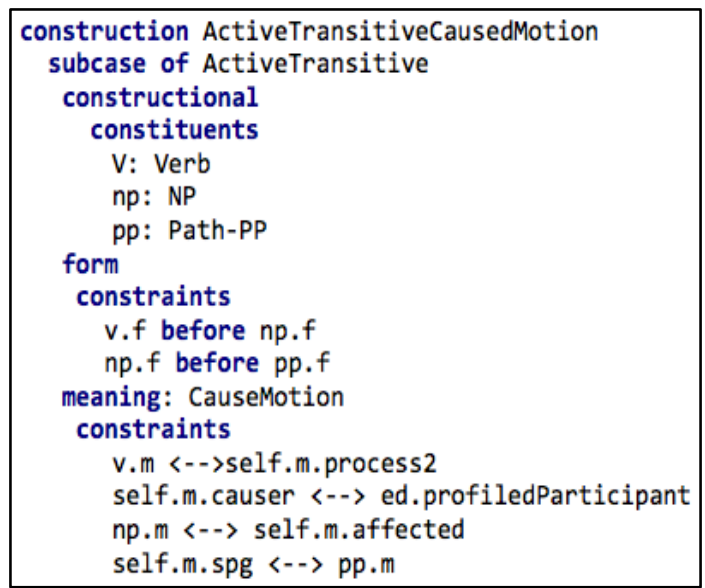

Figure 5:Active_Transitive_Caused_Motion

This is similar to the Active_Motion_Path construction in that it has both a V and a PP constituent. However, as with other A-S constructions that characterize transitives, this A-S construction also includes an NP constituent, whose form follows that of the verb. Crucially, this A-S construction identifies its meaning with CauseMotion, indicating that it is used to describe scenes which a causal agent causes the translational motion of some other entity. Meaning constraints specify that the affected role of CauseMotion is bound to the meaning of the NP constituent, and the causer role is bound to the profiled participant role. This latter constraint indicates that this active voice construction describes caused motion events from the causer's perspective.

Using these constructions, the analysis of Example (2) produces a SemSpec that is similar to that produced for Example (1), with the following key differences:

- the eventProcess is CausedMotion (rather than MotionPath);

- the profiledParticipant role is bound to the causer role of CauseMotion, and to Smedlap;

- $\quad$ the envelope is bound to the affected role of CauseMotion, as well as to the mover role of MotionPath, and the trajector role of SPG.

This SemSpec for Example (2) indicates that:

- the sentence describes an event of caused motion, represented by the CauseMotion schema;

- the caused effect is motion, represented by the MotionPath schema;
- the subject (Smedlap) is the causer of the motion;

- the direct object (the envelope) is the causally affected entity that moves.

- the path of motion is one where with the goal location inside the entity that the prepositional phrase object (the mailbox) specifies, just as in Example (1).

To summarize, the semantic representation output of the ECG analysis process for each of Examples (1) and (2) identifies that they evoke the MotionPath and CauseMotion schemas, respectively (analogous to FN's Motion and Cause Motion, respectively). Also, the output specifies the different roles (FEs) that the different constituents of each sentence realize. Thus, the information provided by such output identifies the frames and frame roles expressed in each sentence.

\section{Extensions}

Given the compositional nature of the ECG grammar, it will also support the analysis of other sentences describing translational motion and caused motion events that differ in terms of the lexical items that are realized. Consider the following new examples.

- His hat is slipping off his head.

- Did the dog roll the ball under the table?

- Which leaf fell off the tree?

Moreover, the approach outlined here clearly also would apply to the analysis of all FrameNet frames that characterize, for instance, cognition, perception, communication or other causal events or transitive actions.

Research has begun to extend the present work to support the analysis of metaphorical uses of motion and caused motion frame structure, as in: The young family slid into poverty or Their huge debts dragged the young family into poverty. This research requires the specification of appropriate constraints on the fillers of the roles that will facilitate distinguishing between the literal and the metaphorical.

\section{References}

J. Bryant. 2008. Best-Fit Constructional Analysis. Ph.D. Thesis, University of California, Berkeley.

N. Chang, S. Narayanan, M. R. L. Petruck. 2002. Putting frames in perspective, Proc. of the $19^{\text {th }}$ COLING, International Conference on Computational Linguistics. Taipei, Taiwan. 
E. K. Dodge. 2010.Constructional and Conceptual Composition Ph.D. Thesis, University of California, Berkeley.

J. Feldman, E. Dodge, J. Bryant. 2010. Embodied Construction Grammar. In B. Heine and H. Narrog (eds.), The Oxford Handbook of Linguistic Analysis, Oxford: Oxford University Press, pp. 111-158.

A. Goldberg. 1995. Constructions: A Construction Grammar Approach to Argument Structure. Chicago: University of Chicago Press.

N. Friedman, M. Linial, I. Nachman and D. Pe'er. 2000. Using Bayesian networks to analyze expression data. Journal of Computational Biology 7.3-4: 601-620.

R. Girju. 2003. Automatic detection of causal relations for question answering, Proceedings of the ACL Workshop on Multilingual Summarization and Question Answering: 76-83.

I. Guyon, A. Elisseeff and C. Aliferis. 2007. Causal feature selection, Computational Methods of Feature Selection Data Mining and Knowledge Discovery Series. Chapman and Hall/CRC, Boca Raton, London, New York, pp. 63-85.

S. Narayanan. 1997. Knowledge-based Action Representations for Metaphor and Aspect (KARMA). Ph.D. Thesis, University of California, Berkeley.

Q. Do, Y. Chan, and D. Roth. 2011. Minimally supervised event causality identification, Proceedings of Empirical Methods in Natural Language Processing (EMNLP), Edinburgh, Scotland, UK.

J. Ruppenhofer, M. Ellsworth, M. R. L. Petruck, C. R. Johnson, and J. Scheffczyk. 2010. FrameNet II: Extended Theory and Practice (Web Publication available via http://framenet.icsi.berkeley.edu). 